OPEN ACCESS

Edited by:

Marcel van der Heyden,

University Medical Center Utrecht,

Netherlands

Reviewed by:

Roddy Walsh,

Academic Medical Center (AMC), Netherlands

Vincent Portero,

Academic Medical Center (AMC),

Netherlands

Tong Liu,

Tianjin Medical University, China

${ }^{*}$ Correspondence:

Carlo Pappone

carlo.pappone@af-ablation.org

tThese authors have contributed equally to this work

Specialty section: This article was submitted to

Cardiac Electrophysiology, a section of the journal

Frontiers in Physiology

Received: 12 February 2019 Accepted: 09 May 2019

Published: 28 May 2019

Citation:

Monasky MM, Micaglio E, Ciconte G, Benedetti S, Di Resta C, Vicedomini G, Borrelli V, Ghiroldi A, Piccoli M, Anastasia L, Santinelli V,

Ferrari M and Pappone C (2019)

Genotype/Phenotype Relationship in

a Consanguineal Family With

Brugada Syndrome Harboring the

R1632C Missense Variant in the SCN5A Gene.

Front. Physiol. 10:666.

doi: 10.3389/fphys.2019.00666

\section{Genotype/Phenotype Relationship in a Consanguineal Family With Brugada Syndrome Harboring the R1632C Missense Variant in the SCN5A Gene}

\author{
Michelle M. Monasky ${ }^{1 \dagger}$, Emanuele Micaglio ${ }^{1 \dagger}$, Giuseppe Ciconte', Sara Benedetti ${ }^{2}$, \\ Chiara Di Resta ${ }^{3,4}$, Gabriele Vicedomini', Valeria Borrelli ${ }^{1}$, Andrea Ghiroldi ${ }^{5}$, Marco Piccoli ${ }^{5}$, \\ Luigi Anastasia ${ }^{5,6}$, Vincenzo Santinelli', Maurizio Ferrari ${ }^{2,3,4}$ and Carlo Pappone ${ }^{1 *}$

\begin{abstract}
'Arrhythmology Department, IRCCS Policlinico San Donato, San Donato Milanese, Milan, Italy, ${ }^{2}$ Laboratory of Clinical Molecular Biology and Cytogenetics, IRCCS San Raffaele Hospital, Milan, Italy, ${ }^{3}$ Genomic Unit for the Diagnosis of Human University, Milan, Italy, ${ }^{5}$ Stem Cells for Tissue Engineering Laboratory, IRCCS Policlinico San Donato, San Donato Milanese, Milan, Italy, ${ }^{6}$ Department of Biomedical Sciences for Health, University of Milan, Milan, Italy
\end{abstract} \\ Pathologies, Division of Genetics and Cellular Biology, IRCCS San Raffaele Hospital, Milan, Italy, ${ }^{4}$ Vita-Salute San Raffaele
}

Brugada syndrome (BrS) is a known cause of sudden cardiac death. The genetic basis of $\mathrm{BrS}$ is not well understood, and no one single gene is linked to even a majority of $\mathrm{BrS}$ cases. However, mutations in the gene SCN5A are the most common, although the high amount of phenotypic variability prevents a clear correlation between genotype and phenotype. Research techniques are limited, as most BrS cases still remain without a genetic diagnosis, thus impairing the implementation of experimental models representative of a general pathogenetic mechanism. In the present study, we report the largest family to-date with the segregation of the heterozygous variant NM_198056:C.4894C>T (p.Arg1632Cys) in the SCN5A gene. The genotype-phenotype relationship observed suggests a likely pathogenic effect of this variant. Functional studies to better understand the molecular effects of this variant are warranted.

Keywords: Brugada syndrome, sudden cardiac death, genetic testing, arrhythmia, SCN5A, sodium channel, cardiomyopathy, variant

\section{BACKGROUND}

The Brugada syndrome (BrS) is diagnosed by the presence of a coved-type ST-segment elevation (type $1 \mathrm{BrS}$ pattern) in the right precordial leads on the electrocardiogram (ECG) that occurs either spontaneously or after administration of a sodium channel blocking agent, such as ajmaline (Antzelevitch et al., 2016), which reveals the type 1 pattern. Patients with this pattern are at increased risk of sudden cardiac death (SCD) (Antzelevitch et al., 2016). The arrhythmias are caused by the presence of an arrhythmogenic substrate (AS) usually found in the epicardial surface of the right ventricle (RV) (Nademanee et al., 2011; Zhang et al., 2015), which can be eliminated by trans catheter radiofrequency (RF) ablation. Ajmaline can be used to fully visualize the extent of the AS and improve the success of the procedure (Pappone et al., 2017). 
The true prevalence of $\mathrm{BrS}$ is currently unknown, as SCD may be the first clinical manifestation in affected individuals. Many patients display a wide degree of clinical variability, even among the same family. BrS is considered as an inherited autosomal dominant disease with incomplete penetrance (Chen et al., 1998; Nademanee et al., 2011; Lieve and Wilde, 2015). Variants in the SCN5A gene are found in about $15-30 \%$ of BrS cases (Kapplinger et al., 2010). SCN5A mutations are found in a number of pathologies, such as arrhythmogenic right ventricular cardiomyopathy, atrial standstill type 1, atrial fibrillation, left ventricular noncompaction, dilated cardiomyopathy, long QT syndrome type 3, sick sinus syndrome type 2, idiopathic ventricular fibrillation, and heart block type 1A (Gosselin-Badaroudine et al., 2014; Zaklyazminskaya and Dzemeshkevich, 2016; Monasky et al., 2018), making it difficult to predict a phenotype from novel variants within SCN5A. In the case of BrS, SCN5A variants are associated with a loss of function of the voltage-gated sodium channel subunit $\left(\mathrm{Na}_{\mathrm{V}} 1.5\right)$ (Di Resta et al., 2015; Sieira et al., 2016; Curcio et al., 2017).

Although a significant enrichment in rare coding variations in patients versus controls was observed only for the SCN5A gene by burden test (Le Scouarnec et al., 2015), genetic mutations are still not detected in the majority of BrS patients (Sonoda et al., 2018), making BrS genetically elusive in many cases. In addition, the importance of genetic background has been highlighted in different studies, questioning Mendelian inheritance and proposing more complex oligogenic models (Bezzina et al., 2013, 2015; Di Resta et al., 2015). Better understanding of the genetics of BrS could assist clinicians in patient risk assessment, especially because ajmaline tests might have a low, but present, false negative rate (Therasse et al., 2017). However, research studies are limited by the lack of understanding of the human genetics in BrS. In the present study, we report a family with a heterozygous variant NM_198056:c.4894C>T (p.Arg1632Cys) in the SCN5A gene. This variant has only been described twice before, once in a single patient with a complex arrhythmogenic phenotype (Nakajima et al., 2015) and once in two siblings with vastly different phenotypes (Garcia-Molina et al., 2016). Thus, the phenotype associated with the variant described herein is uncertain in the current literature. In the present study, we report for the first time the genotype-phenotype correlation in several individuals, providing the strongest evidence to-date as to the possible effects of this variant.

\section{CASE PRESENTATIONS}

The proband is a 32-year-old male of Italian origin with a history of syncope and hypothyroidism treated with substitutive levothyroxine. He performed an arrhythmogenic evaluation after a routine ECG (performed for known hypothyroidism) revealed a type $1 \mathrm{BrS}$ pattern (Figure 1). Thus, the proband underwent electrophysiological study (EPS), which was negative. The patient received ajmaline to reveal the full extent of the AS prior to substrate catheter ablation (Figure 2).

\section{Genetic Studies}

Genetic testing was performed on peripheral blood-extracted genomic DNA with massive parallel sequencing (NGS) using Illumina TruSight Cardio enrichment and MiSeq platform. NGS yielded a coverage $>20 \mathrm{X}$ in $99.6 \%$ targeted regions and an average depth $=279 \mathrm{X}$. Variants were called exploiting both commercial and in-house pipelines based one BWA, SmithWaterman Algorithm, Freebayes, SnpSift-SnpEFF, MiSeq reporter. Sixteen BrS genes were analyzed: CACNA1C, CACNA2D1, CACNB2, GPD1L, HCN4, KCND2, KCND3, MOG1, PKP2, RANGRF, SCN10A, SCN1B, SCN2B, SCN3B, SCN5A, and TRPM4. Variants with a minor allele frequency $>0.01$ according to ExAC database and synonymous or intronic variants if not reported as pathogenic were filtered out.

The NM_198056:c.4894C>T (p.Arg1632Cys) variant identified by NGS in the SCN5A gene was confirmed by Sanger sequencing using standard protocols (Kieleczawa, 2005) and the following primers: Forward_GCACAGTGATGCTGGCTGGAA Reverse_ GCAGAGTGGGGTCGCAGTAGG (Figure 3).

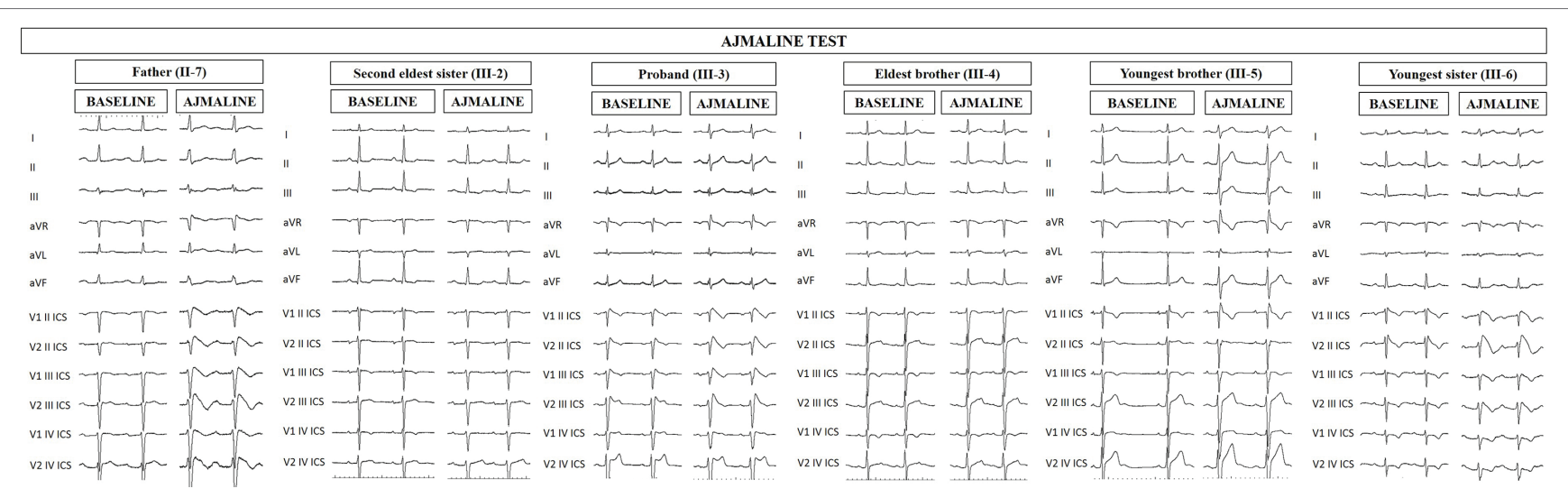

FIGURE 1 | Electrocardiograms of proband and family members at baseline and after ajmaline challenge. Proband's father (II-7), proband (III-3), and proband's youngest sister (III-6) exhibit the BrS type 1 pattern after ajmaline administration, confirming the BrS diagnosis. Proband's second eldest sister (III-2) and brothers (III-4 and III-5) are ajmaline negative. 


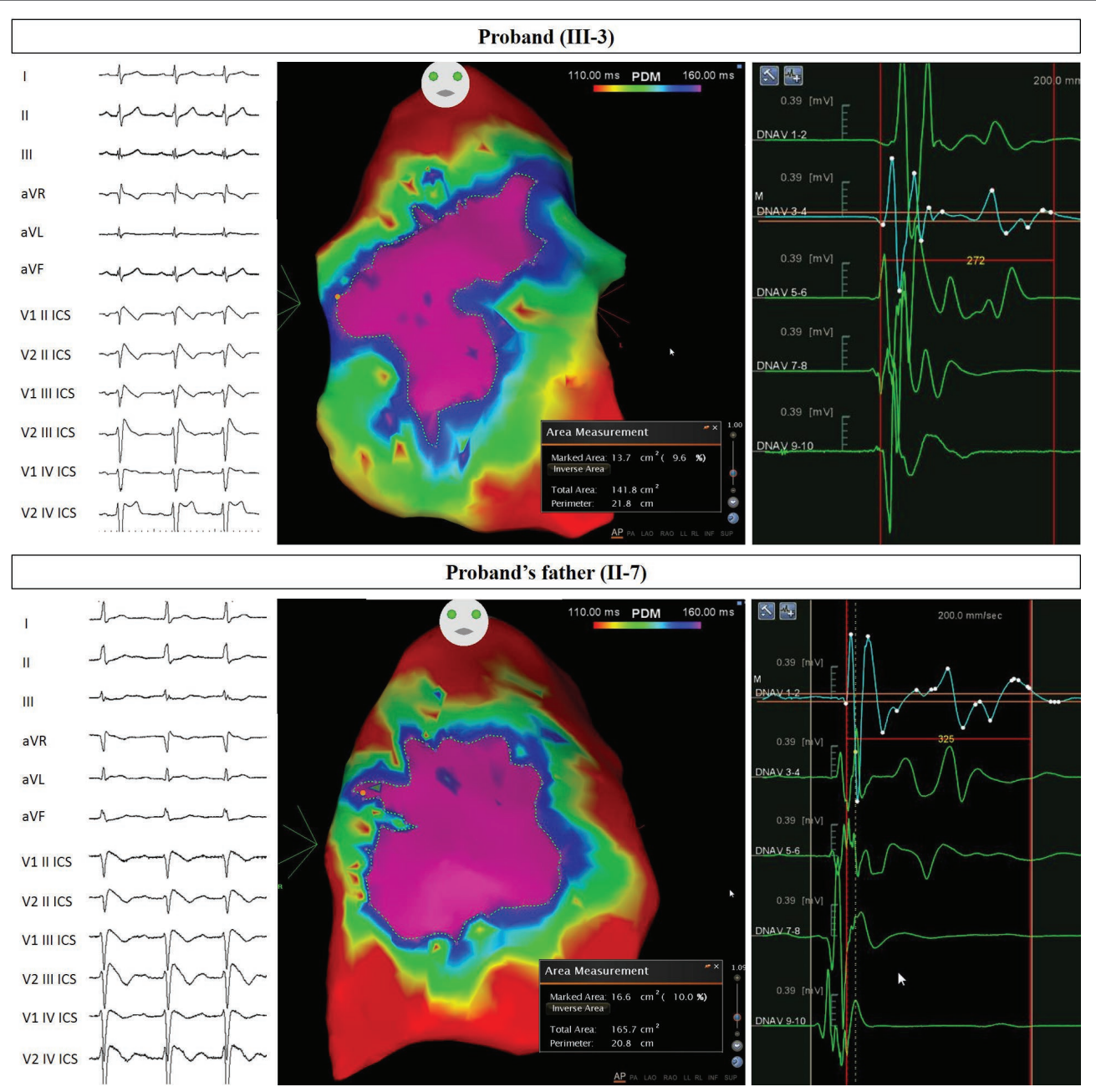

FIGURE 2 | Arrhythmogenic substrate characterization in Proband and Father. Electrocardiogram (left) demonstrates the type 1 BrS pattern after ajmaline administration, confirming the diagnosis. Potential duration map after ajmaline infusion (center) reveals the full extent of the arrhythmogenic substrate. The area of the substrate exhibiting potential durations at least $160 \mathrm{~ms}$ in duration is denoted by the "marked area" and is $13.7 \mathrm{~cm}^{2}$ for Proband and $16.6 \mathrm{~cm}{ }^{2}$ for Proband's father. The duration of fragmented potentials (right) were prolonged and measured 272 ms for Proband and 325 ms for Proband's father.

\section{Assessment of Family Members}

The proband's father is a 64-year-old male diagnosed at the age of 55 with arterial hypertension (pharmacologically treated with ACE inhibitors, successfully) and binodal heart disease. $\mathrm{He}$ came to our attention for familial history of BrS (in his son, the proband: see family tree, Figure 4). He received arrhythmologic evaluation by his family doctor, who recommended ajmaline challenge (Figure 1, Table 1) and an EPS. The results of both of these exams were positive, so an implantable cardioverter device was implanted. After a couple of months, he underwent both binodal disease and BrS arrhythmogenic substrate ablation with a technique based on a transcatheterial application of radiofrequency (Figure 2). Both these procedures were successful and without any operative or postoperative complications. It is interesting that after the two ablations, the arterial hypertension improved, requiring a lesser dosage of antihypertensive drugs that he had taken for the previous 8 years. He underwent genetic counseling and was advised to undergo genetic testing for BrS. This test was performed on peripheral blood-extracted genomic DNA and sought only the heterozygous variant NM_198056:c.4894C >T (p.Arg1632Cys) in the SCN5A gene. This test was positive, confirming a paternal origin for the variant discovered in the proband. The mother had also been genetically tested, due to the consanguinity with her husband, and she was negative for the variant found in her son.

Proband's eldest sister (III-1) is 36 years old and asymptomatic. Her medical history is unremarkable and negative for arterial hypertension and binodal disease. However, she was referred for both ajmaline challenge and EPS due to a family history of BrS. She refused both of these procedures but accepted to undergo genetic testing to seek the heterozygous variant NM_198056:c.4894C>T (p.Arg1632Cys) in the SCN5A gene. The result was positive.

Proband's second eldest sister (III-2) is a 35-year-old asymptomatic individual with an unremarkable medical history. The clinical arrhythmological evaluation was negative for arterial 

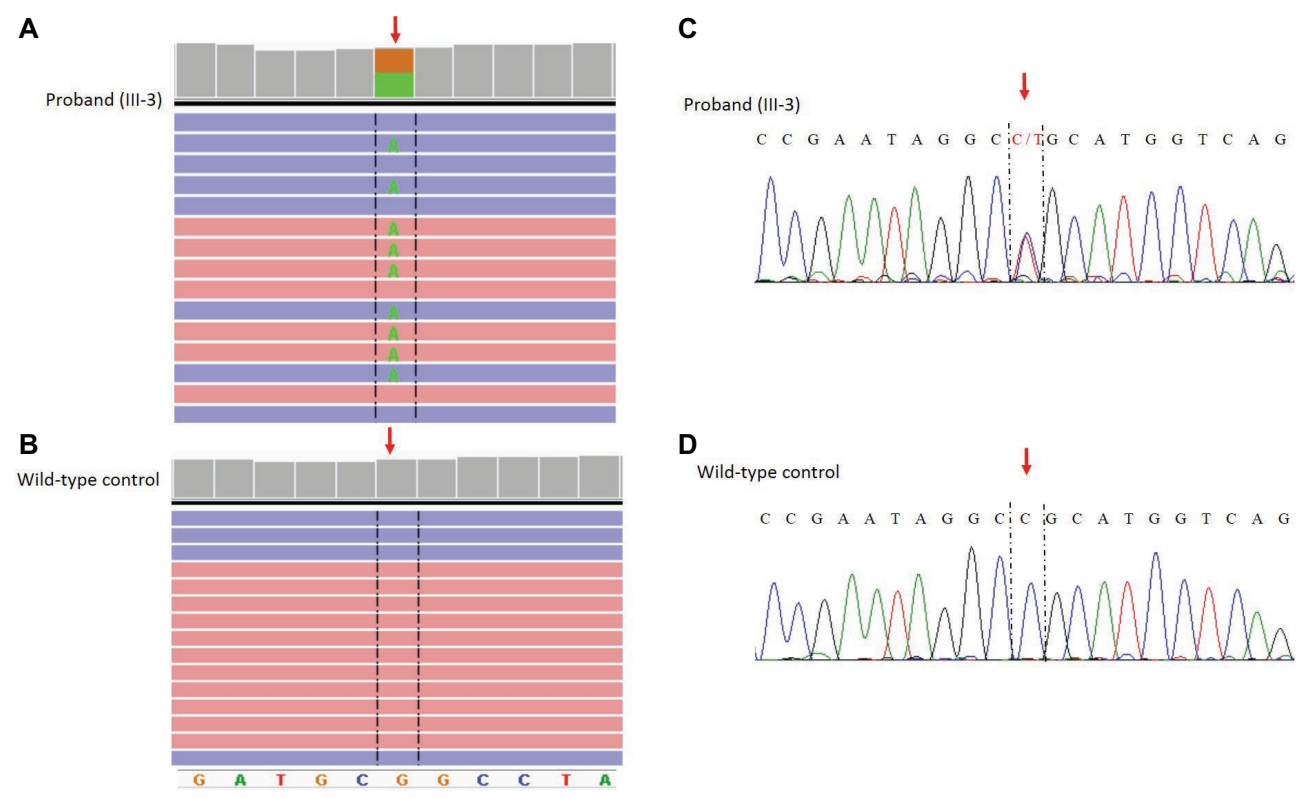

FIGURE 3 | Identification of the c. 4894C>T (p.Arg1632CyS) missense mutation in the SCN5A gene. NGS paired-end reads loaded in the IGV genome browser. The arrow indicates the position of the single nucleotide variation in SCN5A gene in the proband (A) compared to a wild type control sample (B). SCN5A gene is in the reverse orientation on the chromosome. Sanger sequencing electropherogram confirm the presence of the variants in the proband (C) and the absence in a wild type control (D).

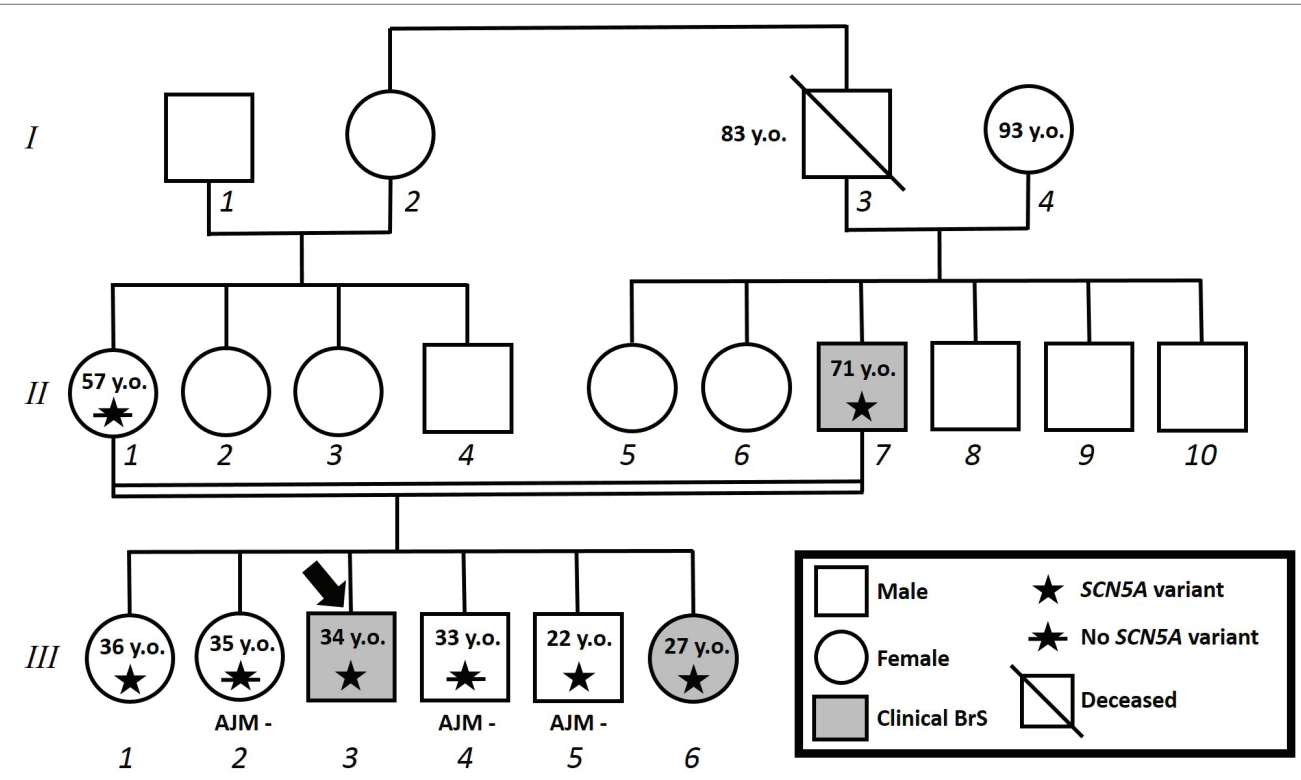

FIGURE 4 | Family pedigree. Proband identified with arrow. Square: male; circle: female; slash: deceased; grayed: clinically affected by Brugada syndrome; star: molecularly confirmed SCN5A variant; star with slash: genetic test for SCN5A variant performed but negative.

hypertension and binodal disease. However, she, like her sister, was referred for both ajmaline challenge and EPS due to family history of BrS. Both of these tests were negative (Figure 1). She also underwent genetic testing to seek the heterozygous variant NM_198056:c.4894C>T (p.Arg1632Cys) in the SCN5A gene, which was negative.
Proband's eldest brother (III-4) is 33 years old and has an unremarkable medical history. The clinical arrhythmological evaluation was negative for both arterial hypertension and binodal disease. However, he, like his siblings, was referred for both ajmaline challenge and EPS due to family history of BrS. Both of these tests were negative (Figure 1), as was 
TABLE 1 | Electrocardiogram parameters at baseline and after ajmaline administration, results of electrophysiological study and genetic testing.

\begin{tabular}{|c|c|c|c|c|c|c|c|c|c|c|}
\hline & \multicolumn{4}{|c|}{ Baseline } & \multicolumn{4}{|c|}{ Ajmaline } & \multirow[t]{2}{*}{ EPS } & \multirow{2}{*}{$\begin{array}{c}\text { SCN5A } \\
\text { variant }\end{array}$} \\
\hline & HR (bpm) & PR (ms) & QRS (ms) & QTc (ms) & HR (bpm) & PR (ms) & QRS (ms) & QTc (ms) & & \\
\hline$\|-7$ & 68 & 290 & 95 & 410 & 72 & 365 & 138 & 510 & Positive & Yes \\
\hline |II-2 & 63 & 186 & 85 & 365 & 69 & 234 & 111 & 401 & Negative & No \\
\hline |II-3 & 88 & 220 & 110 & 420 & - & - & - & - & Negative & Yes \\
\hline |||-4 & 67 & 170 & 107 & 402 & 85 & 222 & 120 & 450 & Negative & No \\
\hline |||-5 & 44 & 181 & 106 & 390 & 59 & 250 & 125 & 445 & Negative & Yes \\
\hline |II-6 & 69 & 244 & 103 & 430 & 75 & 330 & 122 & 485 & Negative & Yes \\
\hline
\end{tabular}

a genetic test for the SCN5A variant found in his family members.

Proband's youngest brother (III-5) is a 22-year-old male affected by allergic asthma, vitiligo, and insulin-dependent diabetes with onset in a pediatric age. No antibodies dosage was available at the time of genetic counseling, but the association with vitiligo suggests an autoimmune pathogenesis (Jin et al., 2007). The patients suffered from recurrent lipothimic episodes, initially thought to be related only to type 1 diabetes. A 12-lead ECG performed because of family history raised the suspicion of BrS. The patient was then referred for EPS, ajmaline challenge, and genetic counseling. The EPS and ajmaline challenge were both negative (Figure 1). Genetic testing seeking the familial variant confirmed this patient harbors the paternally inherited heterozygous variant NM_198056:c.4894C>T (p.Arg1632Cys) in the SCN5A gene. This patient has not performed any genetic testing to assess whether his diabetes could be considered syndromic or not.

Proband's youngest sister (III-6) is 28 years old with a medical history of autoimmune urticaria, autoimmune thyroiditis, and low blood pressure since a pediatric age. She experienced both lypothymic and syncopal episodes during fever and sometimes without fever as well. The arrhythmogenic evaluation performed because of her family history was negative for evidence of major arrhythmias or binodal disease. Considering her father's condition, a 12-lead ECG was performed, which raised the suspicion of BrS. Therefore, an ajmaline challenge was performed and confirmed the BrS diagnosis (Figure 1). An EPS was performed, but the patient was not inducible. This patient was then found to be positive for the same variant in SCN5A that was discovered in her father.

\section{In Silico Predictions and Variant Classification}

Several prediction tools were used to clarify the significance of the SCN5A variant: VarSome genetic database, SIFT, Polyphen2, Mutation Taster, AlignGVGD, and Provean. All of these softwares supported a damaging effect for the p.Arg1632Cys substitution. The Genomic Evolutionary Rate Profiling (GERP) value was noted, which is defined by VarSome as "a conservation score calculated by quantifying substitution deficits across multiple alignments of orthologues using the genomes of 35 mammals. It ranges from -12.3 to 6.17 , with 6.17 being the most conserved" (Cooper et al., 2005). The GERP score for the SCN5A variant described herein was 4.5399. According to ClinVar (2018), no families in which the c.4894C $>\mathrm{T}$ heterozygous variant in the SCN5A gene segregates are provided to date (last update February 18, 2018). The allele frequency is $1: 113,644$ in the general population (2019).

The c.4894C $>\mathrm{T}$ variant was classified as likely pathogenic according to ACMG criteria (Richards et al., 2015):

- PM1: Located in a mutational hot spot and/or critical and well-established functional domain (in this case S4 voltage sensor in domain IV)

- PM2: Extremely low frequency in general population (1/113,644 according to gnomAD) (gnomAD, 2019).

- PM5: Novel missense change at an amino acid residue where a different missense change determined to be pathogenic has been seen before: the p.R1632H was previously reported and determined to affect channel function by functional studies (Benson et al., 2003; Gui et al., 2010).

- PP3: Multiple lines of computational evidence support a deleterious effect on the gene or gene product

- PP1: Cosegregation with disease in multiple affected family members in a gene definitively known to cause the disease

\section{DISCUSSION}

In the present study, we report the genotype-phenotype correlation in the largest family to-date with this heterozygous variant in the SCN5A gene. This study not only underlines the importance of genetic testing to identify family members who require preventive interventions, highlighting its clinical importance.

Since sudden cardiac death may be the first symptom with which a $\mathrm{BrS}$ patient presents, preventive interventions are instrumental in saving these lives. Genetic testing is a noninvasive, and thus safe and painless, method for family member risk stratification. Thus, understanding better the genetics of $\mathrm{BrS}$ not only can help create better disease models but it also has a significant clinical impact.

The SCN5A variant described herein is currently, as of the time of this writing, of unknown significance in the commonly relied upon database VarSome (VarSome, 2018). In silico studies predicted this variant to be damaging, because it is located in a voltage sensitive domain (VSD): a region crucial for the correct function of the $\mathrm{Na}_{V} 1.5$ 
protein (Wang et al., 2016). The activity of the $\mathrm{Na}_{\mathrm{V}} 1.5$ channel protein is controlled by a VSD (Noda et al., 1984) using the positive charges of both lysine and arginine residues. Mutations involving these amino acids have been demonstrated by other groups to reduce the steepness of voltage-dependent gating of the $\mathrm{Na}^{+}$channel. Additionally, these particular kinds of mutations can generate gating pore currents different from the central ionic current, resulting in deep modifications of the $\mathrm{Na}_{\mathrm{V}} 1.5$ gating property (Sottas et al., 2013).

The clinical data in the present study support these in silico predictions and strengthen the hypothesis that this variant is likely pathogenic, as classified according to ACMG criteria (Richards et al., 2015) (see Case Presentations). Additionally, a recently published meta-analysis investigating the prognosis and risk stratification of SCN5A variants in BrS concluded that patients harboring an $S C N 5 A$ variant were at higher risk of arrhythmic events (Yang et al., 2019), supporting our previous findings (Sommariva et al., 2013).

This variant has been previously described in a single patient who experienced a syncopal episode during exercise and who presented with atrial tachycardia, sinus node dysfunction, and BrS (Nakajima et al., 2015). Additionally, the same variant has been described in a brother and sister exhibiting vastly different phenotypes (Garcia-Molina et al., 2016). In that study, a negative $T$ wave and right bundle branch block were observed in the brother, who also tested positive for BrS during a flecainide test. However, the sister was described as a "healthy carrier" who "exhibited a normal baseline and drug challenged ECG." The authors concluded that there was "no evidence that this variant co-segregated with the disease." It is important to note that the authors additionally described two additional variants in the brother, and that the reader is left to assume that the drug challenge performed in the sister was a flecainide test. However, flecainide tests are not as powerful as ajmaline tests in unmasking the type $1 \mathrm{BrS}$ pattern and may result in false negative results (Wolpert et al., 2005).

It is significant to note for this study that the proband and his partner are consanguineous first cousins. Consanguinity increases the risk of inheriting pathogenic variants in both alleles of a disease gene, therefore leading to higher reproductive risk (Saleheen et al., 2017). It seems likely that consanguinity plays a role for arrhythmogenic conditions as well (Al-Hassnan et al., 2017). However, it must be additionally noted that, in spite of the consanguinity, proband's mother tested negative for the familial variant. This finding increases the clinical significance of this variant for the disease that segregates in proband's family. However, consanguinity may lead to the transmission of a modifier SNPs which, when inherited homozygously, may act like a genetic trigger for arrhythmias in symptomatic family members.

One family member (patient III-5) harboring the R1632C variant in the SCN5A gene tested negative during an ajmaline challenge. Type 1 diabetes in this patient may have influenced the outcome of the ajmaline test. Type 1 diabetes causes an insulin depletion that can influence both ST elevation and ST-T wave changes (Nishizaki et al., 2003). Therefore, the
BrS clinical picture can change dramatically as a direct effect of variations in both glucose and insulin concentrations (Velazquez-Rodriguez et al., 2016). However, further studies are needed to better understand the relationship between BrS and diabetes.

\section{CONCLUDING REMARKS}

This study provides the most convincing evidence todate for the genotype/phenotype relationship for the NM_198056:c.4894C > T (p.Arg1632Cys) variant in the SCN5A gene. However, other mechanisms may complicate the genotype/phenotype correlation, including genetic background and particularly the presence of modifier polymorphisms. Further studies in larger groups of patients are therefore warranted to confirm these findings. Genetic testing is a valuable tool to identify at-risk family members and implement preventive interventions. Functional studies are warranted to better understand the molecular pathologies that result from this variant.

\section{DATA AVAILABILITY}

The datasets generated for this study can be found in LOVD, https://databases.lovd.nl/shared/individuals/00222828.

\section{ETHICS STATEMENT}

Written informed consent of human subjects included in this case series report was obtained for their participation in the study and for publication. The procedures employed were reviewed and approved by the local Ethics Committee.

\section{AUTHOR CONTRIBUTIONS}

MM, EM, GC, SB, CR, GV, VB, AG, MP, LA, VS, MF, and $\mathrm{CP}$ collected/analyzed the data. MM and EM wrote the manuscript. All interpreted results, critically reviewed/edited manuscript, and approved final version.

\section{FUNDING}

This study was partially supported by Ricerca Corrente funding from Italian Ministry of Health to IRCCS Policlinico San Donato.

\section{ACKNOWLEDGMENTS}

The authors thank the members of the family for their help and participation in the study. 


\section{REFERENCES}

Al-Hassnan, Z. N., Al-Fayyadh, M., Al-Ghamdi, B., Shafquat, A., Mallawi, Y., Al-Hadeq, F., et al. (2017). Clinical profile and mutation spectrum of long QT syndrome in Saudi Arabia: the impact of consanguinity. Heart Rhythm. 14, 1191-1199. doi: 10.1016/j.hrthm.2017.04.028

Antzelevitch, C., Yan, G. X., Ackerman, M. J., Borggrefe, M., Corrado, D., Guo, J., et al. (2016). J-Wave syndromes expert consensus conference report: emerging concepts and gaps in knowledge. Heart Rhythm. 13, e295-e324. doi: $10.1016 /$ j.hrthm.2016.05.024

Benson, D. W., Wang, D. W., Dyment, M., Knilans, T. K., Fish, F. A., Strieper, M. J., et al. (2003). Congenital sick sinus syndrome caused by recessive mutations in the cardiac sodium channel gene (SCN5A). J. Clin. Invest. 112, 1019-1028. doi: $10.1172 /$ JCI200318062

Bezzina, C. R., Barc, J., Mizusawa, Y., Remme, C. A., Gourraud, J. B., Simonet, F., et al. (2013). Common variants at SCN5A-SCN10A and HEY2 are associated with Brugada syndrome, a rare disease with high risk of sudden cardiac death. Nat. Genet. 45, 1044-1049. doi: 10.1038/ng.2712

Bezzina, C. R., Lahrouchi, N., and Priori, S. G. (2015). Genetics of sudden cardiac death. Circ. Res. 116, 1919-1936. doi: 10.1161/CIRCRESAHA.116.304030

Chen, Q., Kirsch, G. E., Zhang, D., Brugada, R., Brugada, J., Brugada, P., et al. (1998). Genetic basis and molecular mechanism for idiopathic ventricular fibrillation. Nature 392, 293-296. doi: 10.1038/32675

ClinVar. (2018). [Online]. Available at: https://www.ncbi.nlm.nih.gov/clinvar/ (Accessed November 19, 2018).

Cooper, G. M., Stone, E. A., Asimenos, G., Program, N. C. S., Green, E. D., Batzoglou, S., et al. (2005). Distribution and intensity of constraint in mammalian genomic sequence. Genome Res. 15, 901-913. doi: 10.1101/ gr.3577405

Curcio, A., Santarpia, G., and Indolfi, C. (2017). The Brugada syndrome-from gene to therapy. Circ. J. 81, 290-297. doi: 10.1253/circj.CJ-16-0971

Di Resta, C., Pietrelli, A., Sala, S., Della Bella, P., De Bellis, G., Ferrari, M., et al. (2015). High-throughput genetic characterization of a cohort of Brugada syndrome patients. Hum. Mol. Genet. 24, 5828-5835. doi: 10.1093/hmg/ ddv302

Garcia-Molina, E., Sabater-Molina, M., Munoz, C., Ruiz-Espejo, F., and Gimeno, J. R. (2016). An R1632C variant in the SCN5A gene causing Brugada syndrome. Mol. Med. Rep. 13, 4677-4680. doi: 10.3892/ mmr.2016.5100

gnomAD: genome aggregation database. (2019). [Online]. Available at: https:// gnomad.broadinstitute.org/ (Accessed April 17, 2019).

Gosselin-Badaroudine, P., Moreau, A., and Chahine, M. (2014). Nav 1.5 mutations linked to dilated cardiomyopathy phenotypes: is the gating pore current the missing link? Channels (Austin) 8, 90-94. doi: 10.4161/chan.27179

Gui, J., Wang, T., Jones, R. P., Trump, D., Zimmer, T., and Lei, M. (2010). Multiple loss-of-function mechanisms contribute to SCN5A-related familial sick sinus syndrome. PLoS One 5:e10985. doi: 10.1371/annotation/ e86fd0b3-3f5f-4543-a3a1-9004dbale6b5

Jin, Y., Mailloux, C. M., Gowan, K., Riccardi, S. L., Laberge, G., Bennett, D. C., et al. (2007). NALP1 in vitiligo-associated multiple autoimmune disease. N. Engl. J. Med. 356, 1216-1225. doi: 10.1056/NEJMoa061592

Kapplinger, J. D., Tester, D. J., Alders, M., Benito, B., Berthet, M., Brugada, J., et al. (2010). An international compendium of mutations in the SCN5Aencoded cardiac sodium channel in patients referred for Brugada syndrome genetic testing. Heart Rhythm. 7, 33-46. doi: 10.1016/j.hrthm.2009.09.069

Kieleczawa, J. (2005). Simple modifications of the standard DNA sequencing protocol allow for sequencing through siRNA hairpins and other repeats. J. Biomol. Tech. 16, 220-223.

Le Scouarnec, S., Karakachoff, M., Gourraud, J. B., Lindenbaum, P., Bonnaud, S., Portero, V., et al. (2015). Testing the burden of rare variation in arrhythmiasusceptibility genes provides new insights into molecular diagnosis for Brugada syndrome. Hum. Mol. Genet. 24, 2757-2763. doi: 10.1093/hmg/ ddv036

Lieve, K. V., and Wilde, A. A. (2015). Inherited ion channel diseases: a brief review. Europace 17(Suppl 2), ii1-ii6. doi: 10.1093/europace/euv105

Monasky, M. M., Pappone, C., Piccoli, M., Ghiroldi, A., Micaglio, E., and Anastasia, L. (2018). Calcium in Brugada syndrome: questions for future research. Front. Physiol. 9:1088. doi: 10.3389/fphys.2018.01088
Nademanee, K., Veerakul, G., Chandanamattha, P., Chaothawee, L., Ariyachaipanich, A., Jirasirirojanakorn, K., et al. (2011). Prevention of ventricular fibrillation episodes in Brugada syndrome by catheter ablation over the anterior right ventricular outflow tract epicardium. Circulation 123, 1270-1279. doi: 10.1161/CIRCULATIONAHA.110.972612

Nakajima, T., Kaneko, Y., Saito, A., Ota, M., Iijima, T., and Kurabayashi, M. (2015). Enhanced fast-inactivated state stability of cardiac sodium channels by a novel voltage sensor SCN5A mutation, R1632C, as a cause of atypical Brugada syndrome. Heart Rhythm. 12, 2296-2304. doi: 10.1016/j.hrthm.2015.05.032

Nishizaki, M., Sakurada, H., Ashikaga, T., Yamawake, N., Fujii, H., Arita, M., et al. (2003). Effects of glucose-induced insulin secretion on ST segment elevation in the Brugada syndrome. J. Cardiovasc. Electrophysiol. 14, 243-249. doi: $10.1046 /$ j.1540-8167.2003.02389.x

Noda, M., Shimizu, S., Tanabe, T., Takai, T., Kayano, T., Ikeda, T., et al. (1984). Primary structure of electrophorus electricus sodium channel deduced from cDNA sequence. Nature 312, 121-127. doi: 10.1038/312121a0

Pappone, C., Brugada, J., Vicedomini, G., Ciconte, G., Manguso, F., Saviano, M., et al. (2017). Electrical substrate elimination in 135 consecutive patients with Brugada syndrome. Circ. Arrhythm. Electrophysiol. 10:e005053. doi: 10.1161/CIRCEP.117.005053

Richards, S., Aziz, N., Bale, S., Bick, D., Das, S., Gastier-Foster, J., et al. (2015). Standards and guidelines for the interpretation of sequence variants: a joint consensus recommendation of the American College of Medical Genetics and Genomics and the Association for Molecular Pathology. Genet. Med. 17, 405-424. doi: 10.1038/gim.2015.30

Saleheen, D., Natarajan, P., Armean, I. M., Zhao, W., Rasheed, A., Khetarpal, S. A., et al. (2017). Human knockouts and phenotypic analysis in a cohort with a high rate of consanguinity. Nature 544, 235-239. doi: 10.1038/ nature22034

Sieira, J., Dendramis, G., and Brugada, P. (2016). Pathogenesis and management of Brugada syndrome. Nat. Rev. Cardiol. 13, 744-756. doi: 10.1038/ nrcardio.2016.143

Sommariva, E., Pappone, C., Martinelli Boneschi, F., Di Resta, C., Rosaria Carbone, M., Salvi, E., et al. (2013). Genetics can contribute to the prognosis of Brugada syndrome: a pilot model for risk stratification. Eur. J. Hum. Genet. 21, 911-917. doi: 10.1038/ejhg.2012.289

Sonoda, K., Ohno, S., Ozawa, J., Hayano, M., Hattori, T., Kobori, A., et al. (2018). Copy number variations of SCN5A in Brugada syndrome. SCN5A CNVs in BrS. Heart Rhythm. 15, 1179-1188 doi: 10.1016/j.hrthm.2018.03.033

Sottas, V., Rougier, J. S., Jousset, F., Kucera, J. P., Shestak, A., Makarov, L. M., et al. (2013). Characterization of 2 genetic variants of $\mathrm{Na}(\mathrm{v})$ 1.5-arginine 689 found in patients with cardiac arrhythmias. J. Cardiovasc. Electrophysiol. 24, 1037-1046. doi: 10.1111/jce.12173

Therasse, D., Sacher, F., Petit, B., Babuty, D., Mabo, P., Martins, R., et al. (2017). Sodium-channel blocker challenge in the familial screening of Brugada syndrome: safety and predictors of positivity. Heart Rhythm. 14, 1442-1448. doi: $10.1016 /$ j.hrthm.2017.06.031

VarSome: The Human Genomics Community. (2018). [Online]. Available at: https://varsome.com/ (Accessed November 19, 2018).

Velazquez-Rodriguez, E., Rodriguez-Pina, H., Pacheco-Bouthillier, A., and Jimenez-Cruz, M. P. (2016). Efficacy and safety of dextrose-insulin in unmasking non-diagnostic Brugada ECG patterns. J. Electrocardiol. 49, 957-966. doi: 10.1016/j.jelectrocard.2016.08.007

Wang, H. G., Zhu, W., Kanter, R. J., Silva, J. R., Honeywell, C., Gow, R. M., et al. (2016). A novel NaV1.5 voltage sensor mutation associated with severe atrial and ventricular arrhythmias. J. Mol. Cell. Cardiol. 92, 52-62. doi: 10.1016/j.yjmcc.2016.01.014

Wolpert, C., Echternach, C., Veltmann, C., Antzelevitch, C., Thomas, G. P., Spehl, S., et al. (2005). Intravenous drug challenge using flecainide and ajmaline in patients with Brugada syndrome. Heart Rhythm. 2, 254-260. doi: 10.1016/j.hrthm.2004.11.025

Yang, Y., Hu, D., Sacher, F., Kusano, K. F., Li, X., Barajas-Martinez, H., et al. (2019). Meta-analysis of risk stratification of SCN5A with Brugada syndrome: is SCN5A always a marker of low risk? Front. Physiol. 10:103. doi: 10.3389/ fphys.2019.00103

Zaklyazminskaya, E., and Dzemeshkevich, S. (2016). The role of mutations in the SCN5A gene in cardiomyopathies. Biochim. Biophys. Acta 1863, 1799-1805. doi: 10.1016/j.bbamcr.2016.02.014 
Zhang, J., Sacher, F., Hoffmayer, K., O'hara, T., Strom, M., Cuculich, P., et al. (2015). Cardiac electrophysiological substrate underlying the ECG phenotype and electrogram abnormalities in Brugada syndrome patients. Circulation 131, 1950-1959. doi: 10.1161/CIRCULATIONAHA.114.013698

Conflict of Interest Statement: The authors declare that the research was conducted in the absence of any commercial or financial relationships that could be construed as a potential conflict of interest.
Copyright (c) 2019 Monasky, Micaglio, Ciconte, Benedetti, Di Resta, Vicedomini, Borrelli, Ghiroldi, Piccoli, Anastasia, Santinelli, Ferrari and Pappone. This is an open-access article distributed under the terms of the Creative Commons Attribution License (CC BY). The use, distribution or reproduction in other forums is permitted, provided the original author(s) and the copyright owner(s) are credited and that the original publication in this journal is cited, in accordance with accepted academic practice. No use, distribution or reproduction is permitted which does not comply with these terms. 